\title{
EDITORIAL
}

\section{Intracranial pressure thresholds in severe traumatic brain injury: Pro}

\author{
John A. Myburgh ${ }^{1,2,3^{*}}$
}

๑ 2018 Springer-Verlag GmbH Germany, part of Springer Nature and ESICM

Teleologically, the mammalian brain has evolved to be the central component of life. It co-ordinates afferent and efferent neural pathways, integrates neurohormonal responses and, in humans, produces higher cortical effects that augment developmental and protective processes [1]. Despite this vital role for human survival, the brain is profoundly vulnerable to injury and is in many ways set up to fail with devastating consequences for the patient. Central to this thesis is the anatomical and functional relationship of the brain within its protective enclosures and the impact caused by traumatic brain injury (TBI).

Described 300 years ago by Monroe and Kellie, the non-compliant nature of the skull and associated tentorial cavities renders the non-compressible brain susceptible to sharp increases in intracranial pressure (ICP) for small increases in intracranial volume. This inverse relationship defines intracranial elastance, where exhaustion of a critical pressure/volume threshold results in reductions in cerebral blood flow and substrate delivery to a level where irreversible neuronal damage and death ensues [2].

The complexity of elastance exhaustion is compounded by variations in the adaptive capacity of brain compartments; heterogeneity of primary brain injury, secondary ischaemic-hypoxic insults and age-related changes in brain volume [3].

What has perplexed clinicians for decades is how to measure, quantify and modulate this relationship when there is no validated method of measuring intracranial elastance.

The measurement of ICP in TBI has become standard as the principal metric for clinical practice. However, ICP is a surrogate index of intracranial elastance. As with any surrogate measurement, it does not reflect the complexity of a pathophysiological relationship and is subject to measurement inaccuracies and confounding bias [4].

Defining or applying a critical ICP threshold to direct targeted treatment is primarily based on associations between persistent intracranial hypertension and mortality [5]. The validation of an ICP 'dose' or 'burden' has evolved primarily through expert opinion, single-centre observational and cohort studies [6]. There are wide variations in methods of ICP monitoring, with and without the application of multimodal monitoring to define biochemical and metabolic perturbations relating to the ICP 'dose' [7].

The early editions of the Brain Trauma Foundation (BTF) guidelines for the management of TBI presented the limited evidence-base for ICP monitoring, including indications, methods and treatment thresholds $[8$,

\footnotetext{
*Correspondence: jmyburgh@georgeinstitute.org.au

${ }^{2}$ Department of Intensive Care Medicine, University of New South Wales,

St. George Hospital, Gray Street, Kogarah, Sydney 2217, Australia

Full author information is available at the end of the article
}

For contrasting viewpoints, please go to https://doi.org/10.1007/s0013

4-018-5249-y and https://doi.org/10.1007/s00134-018-5251-4.

\section{实


9]. A consistent statement was that there was insufficient high-quality evidence to make level I recommendations for ICP monitoring and therapies to treat intracranial hypertension. Nevertheless, the 2000 edition included a clinical practice guideline describing an algorithm to treat intracranial hypertension, arbitrarily defined as an ICP $>20-25 \mathrm{mmHg}$ and a cerebral perfusion pressure (CPP) $<70 \mathrm{mmHg}$ [10].

The rapid adoption of this algorithm became widespread, perhaps reflecting clinician uncertainty and the lack of clinical practice guidelines for the management of TBI. However, evidence of adverse outcomes, specifically extracranial complications, associated with aggressive targeting of ICP and CPP thresholds emerged, prompting a reassessment of treatment thresholds. The initial treatment algorithm was removed from subsequent editions of the BTF guidelines, pending high-quality randomised controlled trials (RCTs) and consensus statements to inform the revision of treatment thresholds and therapies $[11,12]$.

Designing and conducting an interventional RCT to compare treatment strategies aimed at reducing intracranial hypertension and reducing mortality requires three key elements: (1) the intervention needs to be biologically effective is improving intracranial elastance by reducing both intracranial pressure and volume; (2) the ICP treatment threshold at which patients are randomised needs to be pragmatic and representative of elastance failure at a point where clinical salvage is both safe and effective; (3) the deployment of the intervention needs to be timed to provide the optimal chance of survival. These considerations were integral in the design of two landmark RCTs comparing decompressive craniectomy to current ICP-specific medical therapy.

In the DECRA trial, patients with diffuse TBI were randomised to undergo either decompressive craniectomy or continued second-tier medical therapy when ICP $>20 \mathrm{mmHg}$ for more than 15 mins within a 1-h period, within $72 \mathrm{~h}$ of injury-designed to assess early, pre-emptive decompressive craniectomy in patients with less severe TBI [13].

In the RESCUEicp trial, patients with diffuse and mass lesion-associated TBI with refractory ICP $>25 \mathrm{mmHg}$ for 1-12 $\mathrm{h}$ were randomised to undergo decompressive craniectomy or continued third-tier medical therapydesigned to target decompressive craniectomy in patients with refractory intracranial hypertension-the more usual clinical scenario for this intervention [14].

Both trials reported significant reductions in ICP associated with decompressive craniectomy compared to medical therapy. While there was no significant reduction in mortality observed in DECRA, there was a $22 \%$ reduction in mortality associated with decompressive craniectomy in RESCUEicp. However, both trials reported more "unfavourable" functional outcomes 6 months after injury in patients who underwent decompressive craniectomy.

Both trials addressed the three intervention principles defined above: (1) decompressive craniectomy consistently and effectively reduces ICP, thereby improving intracranial elastance; (2) the two ICP thresholds are widely used by clinicians and recommended by clinical practice guidelines and (3) the intervention was tested at two time-critical periods representing 'real-life' clinical scenarios.

The results of both trials inform both clinical practice and treatment guidelines: decompressive craniectomy effectively improved intracranial elastance in patients with severe TBI and intracranial hypertension at a critical ICP threshold between 15-30 mmHg. However, for those patients who survived both early and late decompressive craniectomy, functional outcome was worse than in patients who underwent medical therapy. This may be explained by cephalad movement of the brain and resultant stretch-induced neuronal injury once the cranium was removed [15].

That a critical ICP threshold exits for patients to survive is not in question. This critical threshold has been revised in the 4th edition of the BTF guidelines to $22 \mathrm{mmHg}$, although this recommendation predated the publication of the RESCUEicp results and is based on lower-quality evidence [16]. On balance, this threshold should be interpreted as the "best" evidence to date to deliver ICP-specific therapies in a relatively safe and effective manner to reduce mortality.

However, what is emerging from new evidence is that reducing ICP below a critical threshold, however defined or recommended, may not be the key determinant in producing the optimal patient-centred outcome-i.e., how the patient survives, feels and functions $[17,18]$.

How clinicians interpret this will depend on whether the clinical imperative is to produce short-term improvements in measured physiological parameters that may reduce mortality or whether a more conservative, expectant strategy may result in better longer-term functional survival, recognising that a substantial proportion of these patients will die.

The consequences of severe disability are prolonged and profound on survivors, caregivers and society and the quality of survival may be more important than reducing mortality. This must be the imperative when interpreting clinical practice recommendations for the treatment of an individual patient. 


\section{Author details}

${ }^{1}$ Intensive Care Medicine, University of New South Wales, Sydney, Australia.

${ }^{2}$ Department of Intensive Care Medicine, University of New South Wales,

St. George Hospital, Gray Street, Kogarah, Sydney 2217, Australia. ${ }^{3}$ Division of Critical Care, George Institute for Global Health, Sydney, Australia.

Received: 14 May 2018 Accepted: 4 June 2018

Published online: 5 July 2018

\section{References}

1. Hofman MA (2014) Evolution of the human brain: when bigger is better. Front Neuroanat 8:15

2. Stern WE (1963) Intracranial fluid dynamics: the relationship of intracranial pressure to the Monro-Kellie doctrine and the reliability of pressure assessment. J R Coll Surg Edinb 9:18-36

3. Saatman KE, Duhaime AC, Bullock R, Maas Al, Valadka A, Manley GT (2008) Classification of traumatic brain injury for targeted therapies. J Neurotrauma 25:719-738

4. Forsyth RJ, Raper J, Todhunter E (2015) Routine intracranial pressure monitoring in acute coma. Cochrane Database Syst Rev (11):CD002043

5. Badri S, Chen J, Barber J et al (2012) Mortality and long-term functional outcome associated with intracranial pressure after traumatic brain injury. Intensive Care Med 38:1800-1809

6. Vik A, Nag T, Fredriksli OA et al (2008) Relationship of "dose" of intracranial hypertension to outcome in severe traumatic brain injury. J Neurosurg 109:678-684

7. Low D, Kuralmani V, Ng SK, Lee KK, Ng I, Ang BT (2009) Prediction of outcome utilizing both physiological and biochemical parameters in severe head injury. J Neurotrauma 26:1177-1182
8. Bullock R, Chesnut RM, Clifton G et al (1996) Guidelines for the management of severe head injury. Brain Trauma Foundation. Eur J Emerg Med 3:109-127

9. The Brain Trauma Foundation, The American Association of Neurological Surgeons, The Joint Section on Neurotrauma and Critical Care. Intracranial pressure treatment threshold. J Neurotrauma 2000;17:493-5

10. The Brain Trauma Foundation, The American Association of Neurological Surgeons, The Joint Section on Neurotrauma and Critical Care. Critical pathway for the treatment of established intracranial hypertension. J Neurotrauma 2000;17:537-8

11. Bratton SL, Chestnut RM, Ghajar J et al (2007) Guidelines for the management of severe traumatic brain injury. IX. Cerebral perfusion thresholds. J Neurotrauma 24(Suppl 1):S59-S64

12. Chesnut RM, BleckTP, Citerio G et al (2015) A consensus-based interpretation of the benchmark evidence from south american trials: treatment of intracranial pressure trial. J Neurotrauma 32:1722-1724

13. Cooper DJ, Rosenfeld JV, Murray L et al (2011) Decompressive craniectomy in diffuse traumatic brain injury. N Engl J Med 364:1493-1502

14. Hutchinson PJ, Kolias AG, Timofeev IS et al (2016) Trial of decompressive craniectomy for traumatic intracranial hypertension. N Engl J Med 375:1119-1130

15. von Holst H, Li X, Kleiven S (2012) Increased strain levels and water content in brain tissue after decompressive craniotomy. Acta Neurochir 154:1583-1593

16. Carney N, Totten AM, O'Reilly C et al (2017) Guidelines for the management of severe traumatic brain injury, fourth edition. Neurosurgery 80:6-15

17. Chesnut RM, Temkin N, Carney N et al (2012) A trial of intracranial-pressure monitoring in traumatic brain injury. N Engl J Med 367:2471-2481

18. Watson HI, Shepherd AA, Rhodes JKJ, Andrews PJD (2018) Revisited: a systematic review of therapeutic hypothermia for adult patients following traumatic brain injury. Crit Care Med 46:972-979 